\title{
Spontaneous Regression of Intraprocedural Carotid Stent Thrombosis Achieved Using Antiplatelet Agents
}

\author{
Jei Kim*1,3, Hyun-Jo Kwon ${ }^{2,3}$ and Jun-Sik Lee ${ }^{1,3}$ \\ ${ }^{1}$ Departments of Neurology, Chungnam National University Hospital and School of Medicine, South Korea \\ ${ }^{2}$ Department of Neurosurgery, Chungnam National University Hospital and School of Medicine, South Korea \\ ${ }^{3}$ Daejeon-Chungnam Regional Cerebrovascular Center, Chungnam National University Hospital and School of Medicine, South Korea
}

\author{
*Corresponding author: Jei Kim, MD, Department of Neurology, Chungnam \\ National University Hospital, 282 Moonhwa-ro, Joong-gu, Daejeon 301-721, South \\ Korea.
}

Received Date: February 26, 2020

Published Date: March 06, 2020

\begin{abstract}
Background and Purpose: The natural process of and treatment plan to prevent intraprocedural carotid stent thrombosis progression have not been clearly described.

Summary of case: We report the case of a 63-year-old male in whom successful regression of an intraprocedural carotid stent thrombus without clinical aggravation was achieved with antiplatelet agent use during and after stent placement.

Conclusion: The present case illustrates the regression process of an intraprocedural carotid stent thrombosis following the administration of antiplatelet agents during and after the intervention.
\end{abstract}

Keywords: Carotid stent; Thrombosis; Regression; Antiplatelet agent

\section{Introduction}

Acute thrombosis is an uncommon complication during carotid stent intervention. 1 In some patients, an intraprocedural carotid stent thrombosis occurs immediately after stent deployment and can lead to a fatal iatrogenic stroke.2 A clear treatment plan to prevent further thrombus progression has not been established to date. We report a patient with successful regression of an intraprocedural carotid stent thrombus without clinical aggravation who received antiplatelet agents during and after intervention.

\section{Case Presentation}

A 63-year-old male arrived at emergency room due to altered comprehension and communication, and motor weakness on the left side of his face and extremities (grade II) that was noted approximately 2 hours before he arrived at the hospital. On the arrival in emergency room, his total National Institute of
Health Stroke Scale score was nine. Magnetic resonance imaging performed at the emergency room revealed an ischemic lesion in the right frontal cortex. Perfusion-weighted imaging showed delayed perfusion in the right parietal and temporal cortices. The patient underwent intra-arterial thrombectomy to restore right hemisphere perfusion.

On initial angiography, the right internal carotid artery was not visualized below its origin. (Figure 1A) After removal of a thrombus using a suction catheter, the occlusion was recanalized, but severe and focal narrowing at the stenotic lesion was still visualized (Figure 1B). A bare metal carotid stent was deployed inside the focal stenotic lesion. Then, a thrombus lodged in the middle cerebral artery was removed by using a suction catheter twice and by single use of a retractable stent through the carotid stent. 

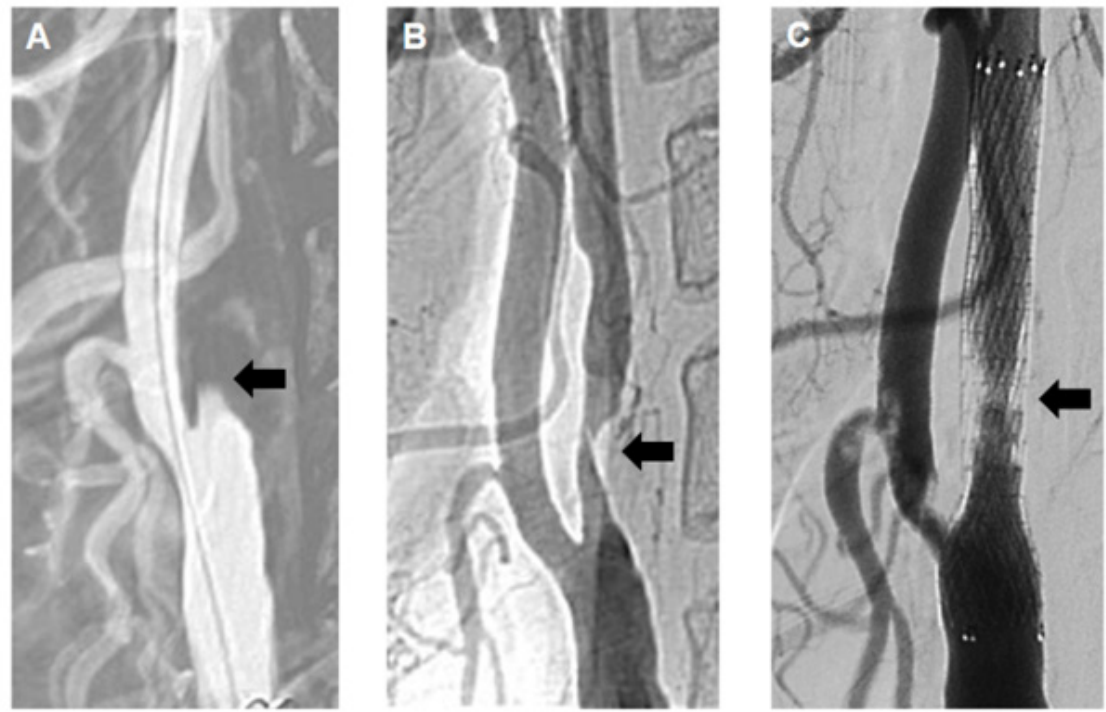

Figure 1: Angiographic images of the right proximal internal carotid artery before and after carotid stent deployment. A right internal carotid artery occlusion (arrow) was observed on initial angiography (A). A focal stenotic lesion (arrow) was visualized after suctioning a thrombus from the site (B). An intraprocedural carotid stent thrombosis (arrow) remained inside the right carotid stent before completing the intervention (C).

Even though right middle cerebral artery recanalization was successful, 66\% stenosis persisted inside carotid stent due to intraprocedural carotid stent thrombus (Figure 1C). To prevent further stenosis progression, a glycoprotein IIb/IIIa antiplatelet agent (tirofiban, $0.5 \mathrm{mg}$ ) was infused inside the carotid stent. The intra-arterial thrombolysis and carotid intervention were completed after no further progression of the thrombus occurred over a 30 -minute period. Immediately after the intervention, the patient received oral aspirin (300 $\mathrm{mg}$ ) and clopidogrel (280 mg). He was maintained on a daily regimen of aspirin $(100 \mathrm{mg})$ and clopidogrel $(75 \mathrm{mg})$. follow-up of magnetic resonance imaging performed 1 day after intra-arterial thrombectomy revealed that only the frontal lobe lesion was denser (Figure 1).

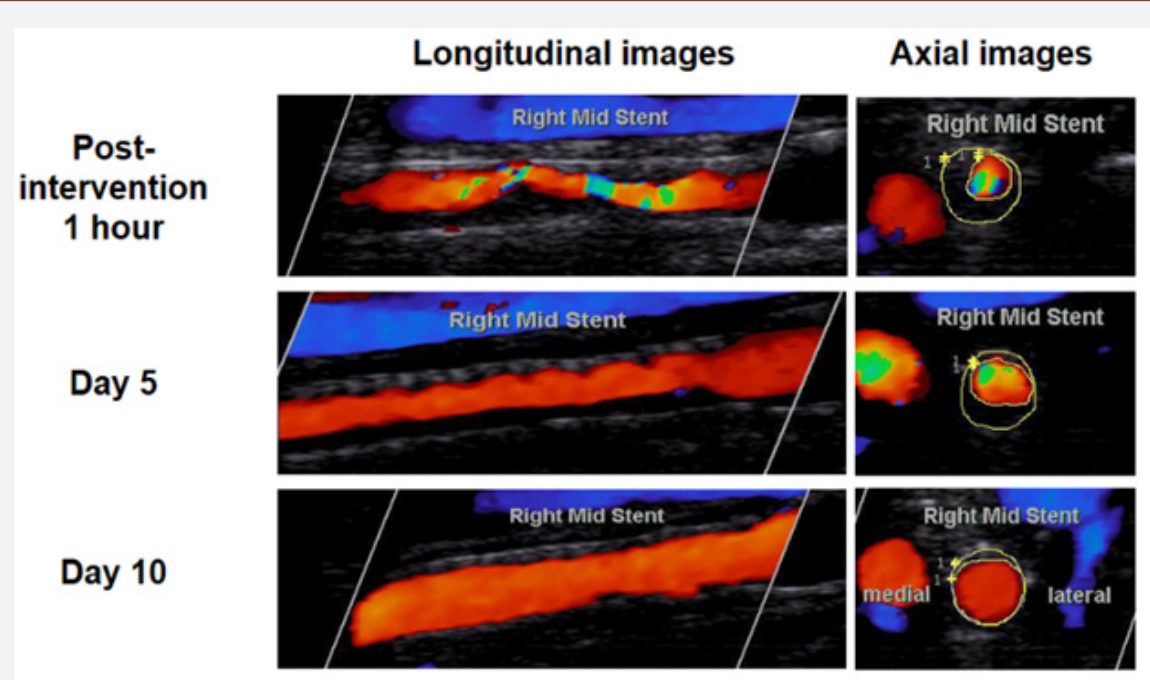

Figure 2: Subsequent changes of intraprocedural carotid stent thrombosis on carotid duplex ultrasonography examinations. One hour postintervention, a diffuse and irregular-margined thrombus narrowed the internal diameter of the right carotid stent $(66 \%, 351 \mathrm{~cm} / \mathrm{s})$. Followup carotid duplex ultrasonography performed 5 days postintervention showed that the thrombus margin was smooth, and the internal diameter was wider $(42 \%, 158 \mathrm{~cm} / \mathrm{s})$. At 10 days postintervention, the narrowing had significantly reduced $(13 \%, 96 \mathrm{~cm} / \mathrm{s})$.

The status of the intraprocedural carotid stent thrombus was examined within 1 hour, daily for next 10 days, and at 17 and 31 days after the intervention using carotid duplex ultrasonography imaging. The first examination performed at 1 hour showed that the thrombus extended through the entire length of the carotid stent and caused acute stenosis $(66 \%$ of stenosis and $351 \mathrm{~cm} / \mathrm{s}$ of blood flow velocity) inside the stent (Figure $2 \& 3$ ). The stenosis severity and blood flow velocity improved on day 2 postintervention. The stenosis reduced to $52 \%(195 \mathrm{~cm} / \mathrm{s})$ and $15 \%(90 \mathrm{~cm} / \mathrm{s})$ at 4 and 10 days after intra-arterial thrombectomy, respectively. The stenosis had decreased to $12 \%(80 \mathrm{~cm} / \mathrm{s})$ at 31 days (Figure $2 \& 3)$. 

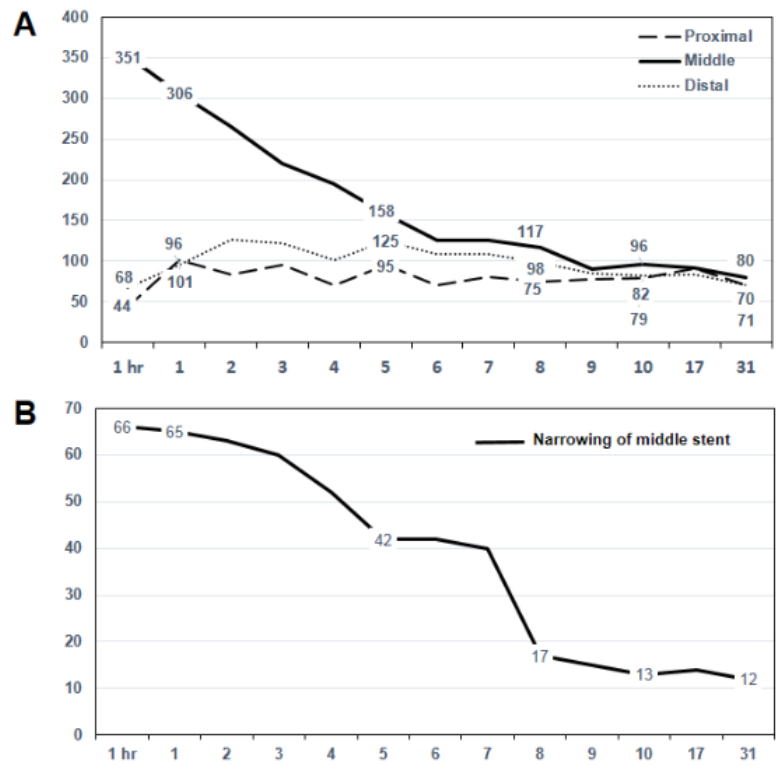

Figure 3: Subsequent changes in blood flow velocity (A) and the narrowed area (B) inside the right carotid stent on carotid duplex ultrasonography follow-up examinations. Blood flow velocity at the middle portion of the inside of the right carotid stent was increased $(351 \mathrm{~cm} / \mathrm{s})$ by the narrow diameter $(66 \%)$. The velocity and narrowed area was subsequently reduced 10 days postintervention. The velocities of the distal and proximal stent regions were decreased 1 hour after the intervention. The next day, the velocity had increased within a normal range, and it decreased over the next 10 days.

Six days after intra-arterial thrombectomy, the patient showed improvement, with partial communication and comprehension and grade III motor weakness in the left extremities. At 31 days, the patient could name items and repeat words in the language function test, and he was able to move his left extremities with grade IV motor power.

\section{Discussion}

The present case demonstrates the 10-day regression process of an intraprocedural carotid stent thrombosis after using intravenous and oral antiplatelet agents. Even though intraprocedural carotid stent thrombosis is a rare complication of carotid stent intervention, it is important to delineate a clear treatment plan to prevent further thrombus progression.

The causes of intraprocedural carotid stent thrombosis are not well understood. In percutaneous coronary intervention, intraprocedural thrombosis occurs in just $0.7 \%$ of patients but is frequently observed on the edge of the plaque. 3 In the present patient, the occlusion by the thrombus also formed on the edge of the carotid plaque, and the intraprocedural carotid stent thrombosis formed inside the carotid stent at the plaque edge. The unstable status of the plaque could have facilitated the formation and progression of the intraprocedural carotid stent thrombus.

The antiplatelet agents aspirin and clopidogrel are often prescribed before and after carotid intervention to prevent thrombus formation inside a carotid stent.4 However, the present patient could not start antiplatelet agents before the intervention because he was initially schedule to undergo intra-arterial thrombolysis to recanalize the right middle cerebral artery. After detection of the intraprocedural carotid stent thrombosis, intravenous antiplatelet agent (glycoprotein IIb/IIIa inhibitor) was administered, and it effectively prevented further thrombus progression during the intervention. Immediate loading and maintenance of two oral antiplatelet agents according to the guideline effectively prevented further progression, and the thrombus regressed without clinical aggravation.

\section{Conclusion}

In conclusion, we described the clinical course of a patient with successful regression of an intraprocedural carotid stent thrombosis achieved with antiplatelet agents. This was a rare case of immediate formation and successful regression of an intraprocedural carotid stent thrombus. The intra- and postprocedural use of antiplatelet agents might be an effective treatment plan to prevent stenosis progression in patients who develop intraprocedural carotid stent thrombosis and have not previously been treated with antiplatelet agents.

\section{Acknowledgement}

None.

\section{Conflict of Interest}

No conflict of interest.

\section{References}

1. Castriota F, Cremonesi A, Tavazzi L (2010) Carotid stenting complications. E-journal of the Cardiology Practice website. E-Journal 8. 
2. Chaturvedi S, Sohrab S, Tselis A (2001) Carotid stent thrombosis: report of 2 fatal cases. Stroke 32(11): 2700-2702.

3. Brener SJ, Cristea E, Kirtane AJ, McEntegart MB, Xu K, et al. (2013) Intraprocedural stent thrombosis: a new risk factor for adverse outcomes in patients undergoing percutaneous coronary intervention for acute coronary syndromes. JACC Cardiovasc Interv 6: 36-43.
4. Brott TG, Halperin JL, Abbara S, Bacharach JM, Barr JD, et al. (2011) ASA/ ACCF/AHA/AANN/AANS/ACR/ASNR/CNS/SAIP/SCAI/SIR/SNIS/SVM/ SVS guideline on the management of patients with extracranial carotid and vertebral artery disease: executive summary. Stroke 42: e420-e463. 